Classification

Physics Abstracts

71.25R $-78.70 \mathrm{D}-07.80$

\title{
Current trends for EELS studies in physics
}

\author{
Philip Edward Batson
}

IBM Thomas J. Watson Research Center, Yorktown Heights, New York 10598, U.S.A.

(Received January 11, 1991; accepted March 26, 1991)

\begin{abstract}
The present level of accuracy and energy resolution attained by parallel recording instruments allows many new details to be extracted from energy loss data obtained from small areas. The new information has inspired theoretical studies to gain further understanding. Contact with the soft x-ray photo-absorption field appears likely to allow better understanding through use of complimentary techniques. Further improvement of the accuracy of the EELS recording equipment is continuing, as well as development of instruments for recording multiple types of experimental data.
\end{abstract}

Electron energy loss scattering (EELS) in the electron microscope has enjoyed a period of steady development during the last twenty years. This period saw the preliminary use of several laboratory built machines [1], a subsequent, rather intense period of theoretical instrumental design [2], the appearance of good quality commercial instruments, and finally array detector recording [3]. Until the parallel array detection became available, experimental work was limited by the very long times required to obtain reasonably accurate data. This could be as long as several hours for a study requiring a few spectra. Therefore, the work was limited to studies in the low loss region, where signals were relatively strong, or to simple elemental microanalysis utilizing a poor energy resolution to improve the collection efficiency. The greatest change brought about by the advent of parallel recording has not been to simply speed up acquisition, but to speed it up by such an extent that it becomes feasible to change the quality and type of information which is routinely available. I will largely limit this discussion to core loss fine structure, as this is the subject that I believe will be most strongly influenced by the quality of the new data. With the new performance, the detailed shape as well as the intensity of core loss signals is available. This has opened a vast set of possibilities, because the shape of core spectra is strongly related to the local atomic bonding environment. Thus we obtain not only the number of atoms of a particular type, but we can say something also about their environment and oxidation state.

At present, a $0.75 \mathrm{eV}$ energy resolution is readily obtainable with commercial instruments. This should be just adequate for studies of bonding in many materials. It does not denote a fundamental limit, however. There is sufficient signal, in principle, to obtain $0.1 \mathrm{eV}$ or better resolution at a core edge in a field emission equipped system. In figure 1 , I show results for the aluminum $L_{2,3}$ edge for the metal and for the $4 \mathrm{~nm}$ amorphous layer of oxide at the metal surface. These spectra were acquired in a few minutes in the Wien filter equipped STEM at IBM. As discussed below, the metal results have been processed to obtain a spectral resolution of $0.16 \mathrm{eV}$. The structure at 
the onset in the metal spectrum is due to spin-orbit splitting and many-body effects. The interest here is that the metal and oxide are readily discernible. Also, the degree of smoothness of the oxide results is not a result of resolution, but a measure of the departure of the oxide from true stoichiometry and structure.

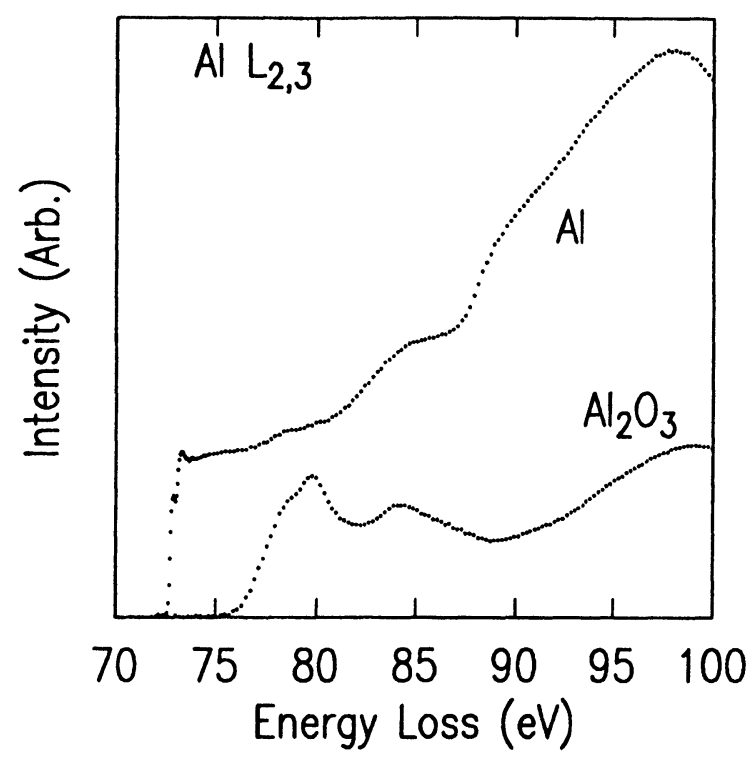

Fig. 1. - Aluminum $\mathrm{L}_{2,3}$ core edges for $\mathrm{Al}$ metal and the oxide. These illustrate the gross differences due to local bonding environment in the soft $\mathrm{x}$-ray absorption region.

In figure 2, I show an example of this behavior by comparing results for silicon, $\mathrm{SiO}_{2}, \mathrm{SiO}, \mathrm{SiO}_{x}$, and $\mathrm{Si}: \mathrm{SiO}_{2}$. The last one is of technological interest for its enhanced carrier injection across the dielectric in the presence of the small silicon islands. [4] In the past, in the absence of imaging detail with which to identify the sub-nm sized islands, there has been no way to differentiate between $\mathrm{SiO}_{x}$, a uniform mixture, and the desired heterogeneous structure of $\mathrm{Si}$ islands embedded in $\mathrm{SiO}_{2}$. In the figure, we can first observe that $\mathrm{SiO}_{2}$ is easily distinguishable from pure silicon. The chemical shift from $99.8 \mathrm{eV}$ to $105 \mathrm{eV}$ is impossible to miss. Examination of the oxide spectra show that there are additional features to show whether the sample is a uniform mixture, and whether it is stoichiometric $\mathrm{SiO}_{2}$ or not, even without reference to the oxygen scattering. In $\mathrm{SiO}_{2}$, either crystalline or good quality amorphous material, the onset consists of a strong exciton peak at $105.7 \mathrm{eV}$, two resonances at 108 and $115 \mathrm{eV}$, and a broad delayed maximum, characteristic of a centrifugal barrier, lying at higher energy. The exciton is strong in the case of the silicon scattering, and weak for the oxygen scattering, consistent with our ideas about charge transfer and resultant screening. The two resonances are the likely result of final state wavefunctions bound within a cage defined by the four oxygen atoms which surround the silicon. Thus, none of the structure in the oxide spectrum appears to be directly relatable to ground state conduction bandstructure. However, "fingerprint" techniques can work very well here. We can see that the "three-peaked" $\mathrm{SiO}_{2}$ structure occurs in all oxide phases. It is likely to be characteristic of the $\mathrm{SiO}_{4}$ bonding unit. It is further suggested then that the $99.8 \mathrm{eV}$ onset energy is characteristic of the $\mathrm{SiSi}_{4}$ unit. From the $\mathrm{SiO}$ spectrum, we can deduce that a uniform sub-stoichiometric oxide exhibits a ramp which 
extends from the oxide excitonic peak smoothly down to $99.8 \mathrm{eV}$ where it disappears. The heterogeneous mixture, $\mathrm{Si}: \mathrm{SiO}_{2}$, however, exhibits a two part spectrum which may be decomposed completely into a silicon part and an oxide part. All this analysis results from the assumption that the scattering occurs in the immediate local environment of an atom. The assumption appears to be valid in enough situations to make the above analysis acceptable. But new theoretical work is required to bring this into routine use.

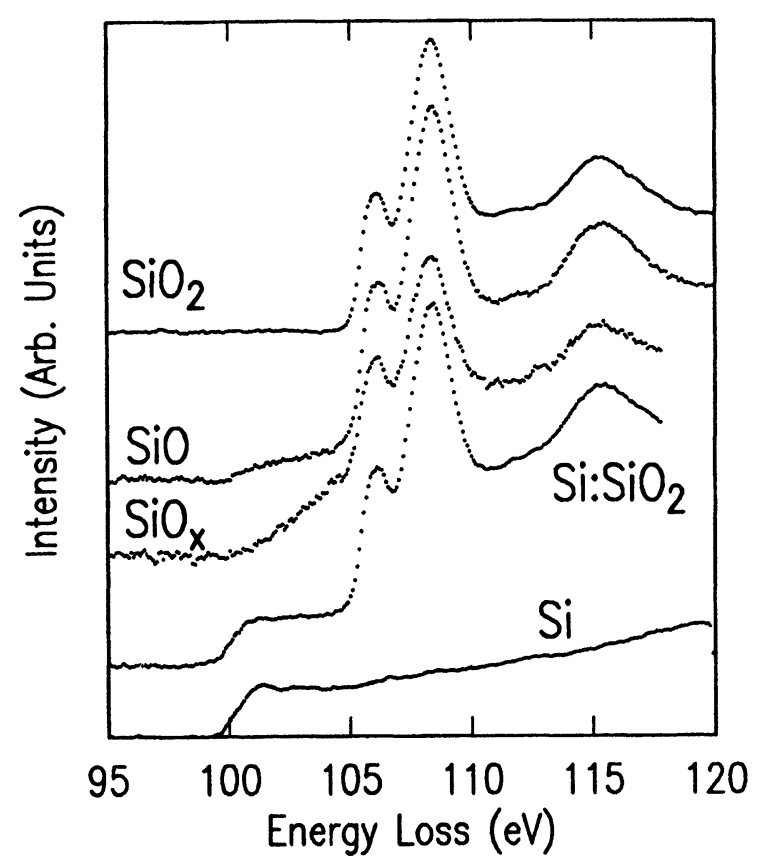

Fig. 2. $-\mathrm{L}_{2,3}$ edges for $\mathrm{Si}$ and its various oxides. It is possible here to discern the difference between a uniform oxide and one containing sub-nm islands of $\mathrm{Si}$, even when no features are observable in the micrographs.

The explosion in information, illustrated by the results in figures 1 and 2, has led to much new work at understanding the shapes of the observed core edges. Much work had been done to understand the basic considerations up into the 1970's [5]. New work, aimed at more convenient evaluation of symmetry-projected densities of states has recently been undertaken. [6] Also, applications of EELS and x-ray photo-absorption (XAS) to materials science problems have inspired theoretical studies of more complicated materials - transition metals and the rare-earth elements for instance [7]. At the present time, it appears that the EELS and XAS efforts are rapidly converging, with the possibility open for interaction and collaboration.

This situation suggests a bright and interesting future for energy loss in the electron microscope. However, discussing the similarities of EELS and XAS techniques does injustice to the probability that the differences between the two will end up being the most interesting. Energy loss in the physical sciences has been largely a tool for investigating the momentum and energy dependence of bulk crystalline solids. We obtain the momentum behavior of an excitation by measuring the intensity variation with scattering angle [8]. An electron microscope is very good at this, and can 
introduce efficiencies over a simple dedicated energy loss machine [19]. But the microscope does not add any new information. New information is obtained when EELS information is obtained together with spatial information. This can be occur in two distinct ways.

First, most simply, we find that EELS spectra are bulk-like but different in adjacent areas of heterogeneous materials. Thus, we obtain spectra which are interpretable as in the bulk, but which are characteristic of sub-micron sized regions of materials. The surprise that has arisen with this operation is that the size of the region can be very small while still displaying bulk properties. For instance, absorption of light in aggregates of small particles by excitation of dipolar surface plasmons has been observed for many years. In fact, this became such a reliable result that it was inferred that sub-micron sized particles are capable of supporting only surface modes which have dipole symmetry. When EELS is obtained in the same systems of particles, using well collimated beams to obtain a good angular resolution, similar results are obtained. However, when the scanning transmission electron microscope (STEM) is used to obtain EELS results from sub-nm sized regions of sub-micron particles, the surface plasmon modes do not normally show dipole symmetry. Rather, short wave-length, higher energy modes prevail, propagating on the particle surface as though it were a large object having a large radius of curvature [10]. It emerges that small particles are entirely capable of supporting non-dipolar excitations, but that excitation by light, or by small angle EELS, does not excite the particles with an applied field having an appropriate non-dipolar symmetry. EELS in the electron microscope, then, can be used to excite small regions of materials with an applied field having a wide range of spatial frequencies to compliment the dipole symmetry available from the optical techniques.

Secondly, EELS in the electron microscope can obtain qualitatively different information. When a coherent electron probe size becomes so small that the transverse momentum uncertainty in it dominates the angular spread of the measurement, we may speak of spatially resolved electron energy loss scattering, SREELS. This can lead to qualitatively different physics, and is the experimental configuration which is most appropriate for add ressing questions regarding electronic structure of defects, bonding at interfaces, and local environment in sub-nm sized regions of materials. In this regime, an occupied defect state can be thought of as a wavepacket consisting of a sum of crystal Bloch states. The high energy electron in the microscope can also be characterized as a wavepacket. Ultimately, then, SREELS will be properly described by a collision of these two wavepackets. In the past, we have thought in terms of simple waves - very parallel electron beams, single crystal Bloch states, and a scattering formulation dependent only on the magnitude and sometimes direction of the transferred wavevector. In the future, it will be more appropriate to think in terms of wavepackets - the coherent probe, a small crystal defect, and a scattering formalism which depends on the complicated nature of localized fields applied to the system. The beginnings of this sort of treatment were introduced during the past ten years with the concept of a "mixed" dynamical form factor. [11] A logical outcome of this way of thinking is that, once spatially resolved spectrometer systems are capable of $\mathrm{meV}$ resolution, inelastic scattering via phonon generation will be properly described as acoustic or thermal wavepacket generation. High frequency mechanical properties of very small volumes may then become a legitimate subject for study by SREELS.

Thus, there are two types of activity which are going to be important in the near future. Categorization of "fingerprints" of various bonding environments will be immediately useful. At the same time, this categorization should lead to better understanding of the systematic variations of the shapes of edges. Secondly, we need a better understanding of the fundamental scattering. How does the detailed shape of a core edge relate to the conduction bandstructure of the solid? Do fundamental assumptions about the angle-resolved scattering geometry and $x$-ray absorption also apply to the spatially-resolved experiments? How does the nanometer scale morphology of the specimen affect the EELS results? 
At first glance, these questions may not seem too relevant, because there has not been good evidence that there is anything wrong with our current understanding of EELS results. For instance, $x$-ray spectra obtained in a spatially resolved soft x-ray absorption instrument [12] are not observably different from those of figure 2 above. In figure 3, I show an expanded plot of the Al near threshold region from figure 1 , compared with x-ray absorption results [13] at the $0.16 \mathrm{eV}$ resolution level. The $\mathrm{Al}$ metal results have been processed to remove the instrumental energy distribution due to the field emission tip. This takes advantage of the fact that the field emission distribution has a relatively sharp high energy onset. The improvement in instrumental response is illustrated by the inset which summarizes the measured instrumental resolution compared with the final processed spectral resolution. It should be remarked in passing that this result is still limited by the instrument, because the intrinsic core level lifetime for soft x-ray edges out to a few hundred $\mathrm{eV}$ give a broadening of order $0.1 \mathrm{eV}$. There are no discernible differences between the EELS and XAS results. On the other hand, figure 4 shows results for diamond, compared with partial photo-yield data [14] and with the p-DOS calculation. [15] Both results closely follow the calculations in the $294-310 \mathrm{eV}$ range. But near threshold, the shape is dominated by transitions to the core exciton. The small peak at the edge is an excitonic bound state, and the intensity in the $289-293 \mathrm{eV}$ range is distorted. The shape can be adequately explained within a simple effective mass theory originally proposed by Elliott. [16] There are clear problems with comparison of the XAS and EELS data in the near-threshold region. Beyond about $294 \mathrm{eV}$, these problems disappear, leaving spectra which are virtually indistinguishable. There are lots of possibilities for breakdown of the comparison. The first is the dipole limit. For small scattering angles, the EELS results should be identical to the XAS results, given by the dipole matrix element between the intitial and final states of the material. In pratice, typical EELS apertures lead to scattering angles corresponding to maximum momentum transfers of $2.0 \AA^{-1}$. Thus, $\mathbf{q} \cdot \mathbf{r} \sim 0.2$ for these edges. We expect the dipole limit to hold under for this condition. The difference between the aluminum and diamond cases is the lack of an exciton for aluminum. On the other hand, $\mathrm{the}^{\mathrm{SiO}_{2}}$ data, where an exciton also is strong, show good correspondence with the XAS results. In other work, I have shown that all of these comparisons can be understood by taking into account the fact that the sudden limit for inelastic electron scattering may be inadequate when the excited state is not characterized very well by the localized crystal wavefunctions. Thus the weak exciton in the case of diamond has a $6 \AA$ radius. Within this radius, the excited core electron resides in a highly distorted state, a wavepacked composed of several crystal wavefunctions. In diamond, this wavepacket is large enough to produce a non-ideal interaction with the high energy electron. In $\mathrm{SiO}_{2}$ the exciton orbit is small, allowing the sudden limit to be realized. Preliminary work [17] to be published elsewhere, shows that this view can be applied to a range of materials.

Thus, there is much theoretical work to be done on the scattering theory to allow an understanding of the core loss results that parallel recording systems are capable of giving us. This is true in the low loss region as well. As discussed elsewhere in this proceedings, there does not exist a general method for extracting bulk dielectric behavior when the sample consists of a heterogeneous mixture of different materials. It appears promising, though, that some type of effective medium theory may help enormously in this task [18].

Once the fundamental scattering is understood, calculations of projected densities of states can explain major features of the near edge fine structure. Figure 5 shows results for Si complared to a calculation for the s, d-projected DOS. The Si results were obtained in a relatively thick sample. The raw data was sharpened to about $0.2 \mathrm{eV}$ resolution, and the $\mathrm{L}_{3}$ edge sharpe was extracted by a fourier analysis technique assuming a spin orbit splitting of $0.608 \mathrm{eV}$ and an occupation weighting of $2: 1$. The extent of agreement is gratifying considering the fact that the matrix elements are considered to be constant in this calculation [19]. Some of the differences near the edge can be 


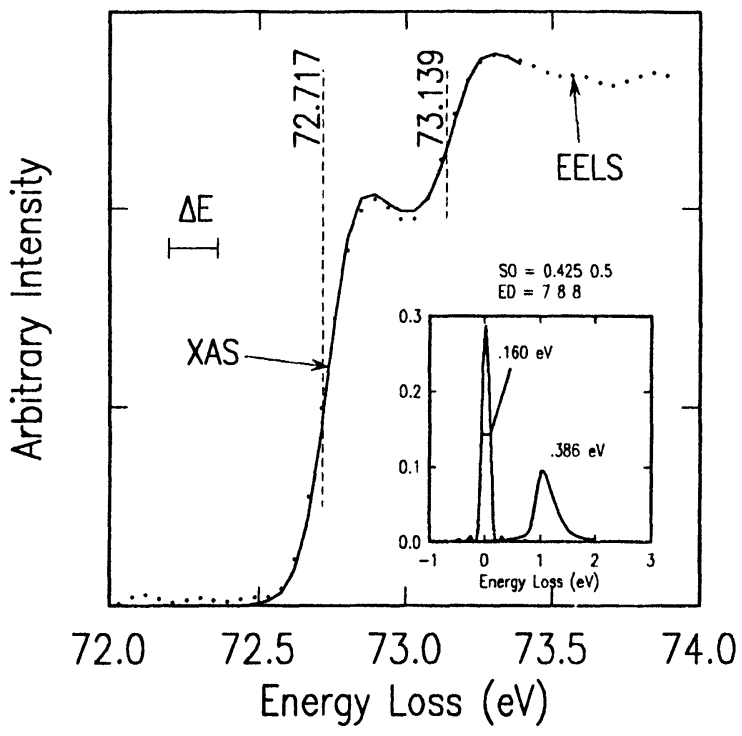

Fig. 3. - $\mathrm{Al} \mathrm{L}_{2,3}$ edge in the region at onset, compared to the $\mathrm{x}$-ray absorption results. The EELS data have been sharpened by unfolding the field emission distribution to attain a spectral resolution of $0.16 \mathrm{eV}$. The inset illustrates this improvement.

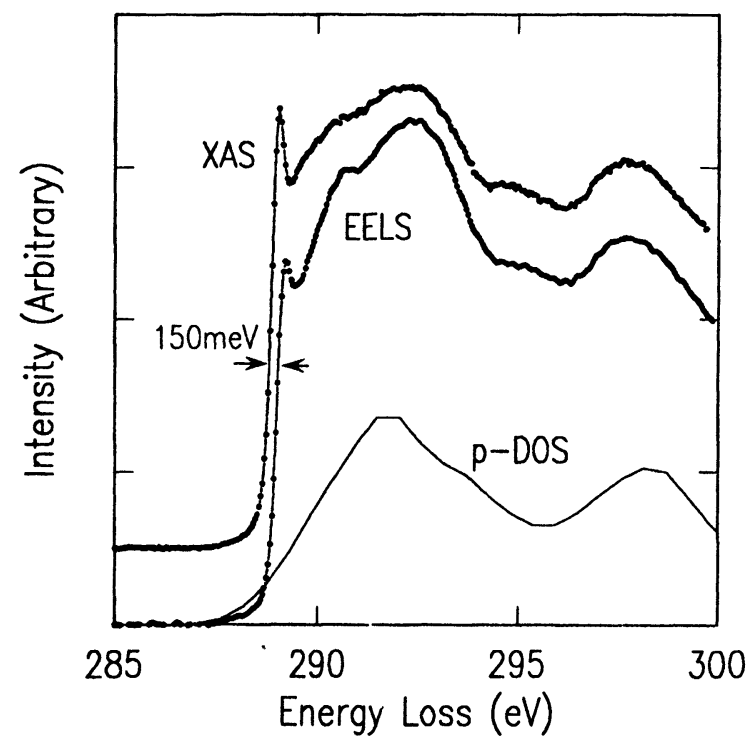

Fig. 4. - Comparison of the carbon $\mathrm{K}$ edge in diamond obtained by EELS and $\mathrm{x}$-ray absorption. The results are different in the region influenced by the core exciton.

attributed to core excitonic distortion, but the magnitude of this distortion was found in this work to be much less than had been assumed in the past.

In this discussion, we have seen that the fundamental scattering theory needs to be reevaluated for some scattering geometries. But in spite of this, detailed calculations of the bandstructure 


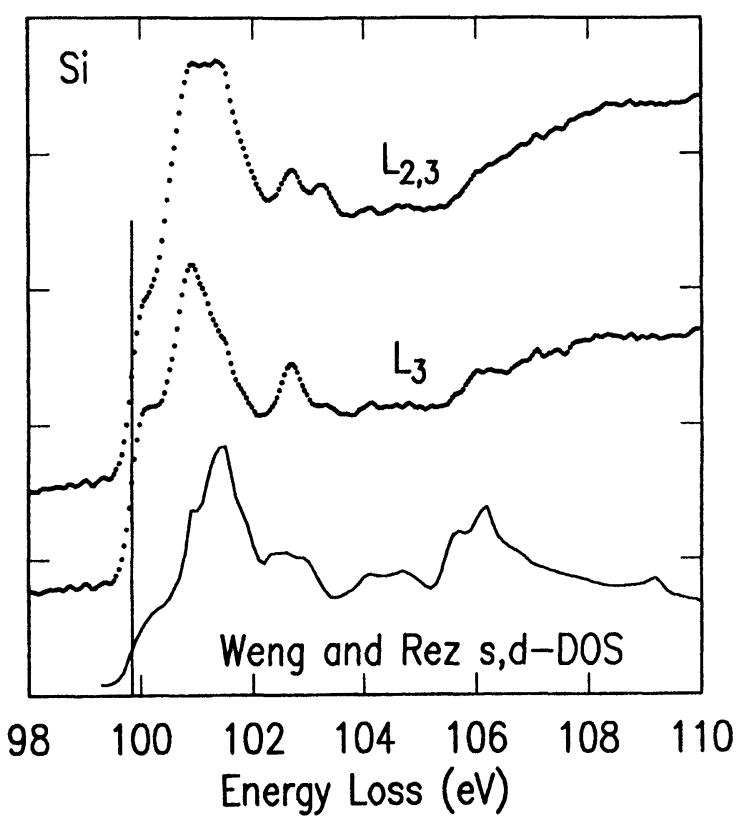

Fig. 5. $-\mathrm{Si} \mathrm{L}_{2,3}$ edge for bulk silicon, compared to the $\mathrm{s}$, d-projected DOS. The $\mathrm{L}_{3}$ results are obtained from a spin-orbit decomposition to display only the part due to the final state. The $L_{2,3}$ data have been sharpened to obtain a resolution of $0.25 \mathrm{eV}$.

and expected spectra for complicated materials are beginning to be successful. In the future, we can expect EELS instruments to gain, perhaps, a factor of ten in collection efficiency and energy resolution. We can expect to see time resolved experiments which trade energy resolution for short recording times. These have already been tried in experimental investigations of radiation damage. We can expect to see more channels of parallel recorded data to be available from our instruments. For instance, inclusion of an array imaging detector can allow parallel measurement of both energy loss and say, scattering angle, or specimen position. We can expect to see more and more results which are not easily explainable in the standard theory. At the same time, theoretical work should begin to tie the already established photon-absorption techniques together with the EELS results to exploit complimentary strengths. The major difference between the past ten years and the next is going to be the availability of very good quality data. This should allow much more detailed and meaningful tests of the conditions under which are theoretical understanding remains valid. Once this has been accomplished, we should be able to unravel truly microscopic characteristics of materials - bandstructure of individual defects, bonding in sub-nanometer clusters, electron gas dynamical behavior within confined volumes, to name a few.

\section{Acknowledgements.}

I want to thank P. Rez, X. Weng, and J. Bruley for significant contributions to this work. 


\section{References}

[1] Metherell A. J. F. and Whelan M. J., J. Appl. Phys. 37 (1966) 1737 ;

CurTIS G. H. and SilcoX J., Rev. Sci. Inst. 42 (1971) 630.

[2] EGERTON R. F., Ultramicr. 3 (1978) 39 ;

SCHEINFEIN M. and ISAACSON M., in "Scanning Electron Microscopy, Part. 4, (SEM, Inc.) 1984, p. 1681 ;

KRIVANEK O. L. and SWANN P. R., in "Quantitative Microanalysis with High Spatial Resolution", (The Metals Society) 1981, p. 136 and BATSON P. E., Rev. Sci. Inst. 57 (1986) 43.

[3] Johnson D. E., CSILlag S., MOnson K. L. and Stern E. A., in 39th Ann. Proc. Electron Microsc. Doc. Am., G. W. Bailey Ed., Claitors, Baton Rouge) 1981, p. 370 ;

EGERTON R. F., J. Electron. Microsc. Tech. 1 (1984) 37 ;

KRIVANEK O. L., AHN C. C. and KEENEY R. B., Ultramic. 22 (1987) 103;

BATSON P. E., Rev. Sci. Inst. 59 (1988) 1132.

[4] Dori L., Bruley J., Di Maria D., Batson P. E., Tornello J. and ARIEnzo M., J. Appl Phys., in press.

[5] INOKUTI M., Rev. Mod. Phys. 43 (1971) 297;

PINES D. and NOZIERES P., Theory of Quantum Liquids, V.I, (Benjamin) 1966, p. 223.

[6] WENG X., REZ P. and SANKeY O. F., Phys. Rev. B40 (1989) 5694.

[7] De GroOt F. M. F., Fuggle J. C., ThOle B. T. and SAWATZKY G. A., Phys. Rev. B42 (1990) 5459.

[8] GibBons P. C., RitsKo J. J. and SchNATTERLEY S. E., Rev. Sci. Inst. 46 (1975) 1546.

[9] BATSON P. E. and SIlcoX J., Phys Rev. B27 (1983) 5224.

[10] BATSON P. E., Surf. Sci. 156 (1985) 720;

RITCHE R. H. and HOWIE A., Phil. Mag. A58 (1988) 753.

[11] KOHL H., Ultramic. 11 (1983) 53.

[12] HARP G. R. and TONNER B. P., Phys. Scr. V. T31 (1990) 23.

[13] OLSEN C. G. and LYNCH D. W., Sol. St. Comm. 31 (1979) 601.

[14] MORAR J. F., HimpSel F. J., HOllinger G., Hughes G. and JoRdan J. L., Phys. Rev. Lett. 54, (1985) 1960.

[15] WENG X., Rez P. and MA H., Phys. Rev. B40 (1989) 4175.

[16] Elliott R. J., Phys. Rev. 108 (1957) 1384.

[17] BATSON P. E. and BRULEY J., Phys. Rev. Lett., to be published.

[18] WALIS M. G. and HOWIE A., Ultramic. 28 (1989) 40.

[19] WENG X., REZ P. and BATSON P. E., Sol. Stat. Comm. 74 (1990) 1013. 